\title{
RESEÑAS BIBLIOGRAFICAS
}

\author{
Clark, Robert L. y Spengler, Joseph J., The economics of individual and po- \\ pulation aging, Cambridge, Cambridge University Press, 1980, págs. viii \\ +211 .
}

La presente obra forma parte de la serie Cambridge Surveys of Economic Literature. El objetivo fundamental de estas publicaciones es el de contribuir a que, tanto los economistas como los estudiantes próximos a graduarse, puedan mantenerse actualizados, especialmente en aquellos campos cuyo contenido va transformándose y cuya literatura se multiplica rápidamente.

En el caso particular de "The economics of individual and population aging", el propósito de los autores es el de identificar y describir los principales temas asociados con el envejecimiento de una población y determinar, así mismo, el estado actual del conocimiento en este campo. centrando el interés en la bibliografía relacionada, total o esencialmente, con los aspectos económicos del envejecimiento, sin dejar de tener presente el carácter interdisciplinario que reviste el problema. Si bien el origen de la investigación relevante se remonta a principios de la década de 1920, la atención de Clark y Spengler se dirige principalmente a la bibliografía publicada desde 1950 en los países industrializados que son, por otra parte, los que soportan más intensamente el proceso del envejecimiento de sus poblaciones.

El libro está conformado por diez capítulos. En el primero, a modo de introducción, los autores destacan la actualidad del tema; definen claramente el proceso del envejecimiento de una población (incremento relativo del grupo de personas de 60-64 años y más); resaltan la relevancia del problema de la dotación de un sistema colectivo de asistencia a la vejez y terminan con la presentación de los temas tratados. Los ocho capítulos siguientes constituyen el cuerpo de la obra donde se tratan, de forma equilibrada. los diversos aspectos del tema: el envejecimiento de la población y sus determinantes; el problema de la dependencia; el status económico de la población de edad más avanzada; la asignación del tiempo en relación a las actividades económicas: la oferta laboral de los trabajadores de mayor edad; las características individuales y de mercado que afectan el retiro; los sistemas de seguridad social y la incidencia, a nivel agregado. del cambio de la estructura por edades. El último capítulo realiza un balance del problema del envejecimiento en relación a su asistencia. La obra incluye una extensa lista de fuentes bibliográficas por capítulo; es de destacar la importancia relativa de esta parte, aproximadamente un $20 \%$ del total de páginas, hecho válido si se tiene en consideración la naturaleza de la obra cual es la de reseñar "las investigaciones realizadas en todo el mundo por economistas, demógrafos y otros investigadores sociales" (pág. vii).

El capítulo 2 considera las determinantes del envejecimiento (fecundidad, expectativas de vida. movimientos migratorios) en relación con la estructura por edades y resalta la importancia 
esencial que la fertilidad reviste en este proceso; presenta también la tendencia del envejecimiento, sus límites y sus proyecciones. En el capítulo 3 se reseña la bilbiografía sobre la dependencia (proporción de la población no activa, con especial referencia a la de mayor edad, sobre el total de activos); el costo que implican los sistemas jubilatorios colectivos y cómo y en qué medida tal carga se ve afectada por cambios en la estructura por edades. A continuación (cap. 4), los autores realizan un resumen crítico de trabajos referentes a la situación económica de las personas de edad avanzada en los países industrializados, con especial referencia a EE. UU., enumeran las fuentes de ingresos de este sector de la población destacando la seguridad social como la de mayor importancia; consideran el impacto de la inflación en el deterioro de su bienestar y otros factores determinantes del status económico de la vejez como son la productividad, la participación en la fuerza laboral, etc. La bibliografía analizada en el capítulo 5 se refiere a las relaciones entre la edad, considerada en los sucesivos estratos, y los aspectos económicos como son la productividad y la capacidad laboral; se destaca la utilidad de la división por grupos de edades tanto para el diseño de políticas socio-económicas como para la teoría moderna sobre la asignación del tiempo que eligen los individuos para dedicarlo a su educación, entrenamiento laboral, trabajo, consumo, inversión; finalmente, trata la manera en que afectan a las personas de los últimos estratos de edad, las decisiones de los grupos restantes en cuanto a actividades y planes. El capítulo 6 está dedicado a estudios referidos a la participación de las personas en edad avanzada en la oferta laboral; a los factores que influyen sobre la decisión de permanecer en el mercado de trabajo; a la aplicación del modelo básico de oferta de mano de obra a esta cohorte; a las dificultades que presenta el tratamiento de las fuentes estadísticas de la oferta de empleo y retiro en las distintas actividades por tratarse de un grupo heterogéneo influenciado por una variedad de factores socio-económicos; finalmente, al impacto que sobre la decisión del retiro representan los sistemas de seguridad social y otras fuentes suplementarias de ingresos y la posibilidad de elegir la edad de jubilación en estrecha compatibilidad con el envejecimiento. El retiro también es el tema del capítulo 7 ; en este caso, se trata en relación a las características personales y de mercado de la fuerza de trabajo, tales como la salud, el nivel de empleo de la economía, el proceso de urbanización, los salarios, la ocupación, para señalar los más significativos; se confrontan las posturas de distintos autores sobre la preminencia que, desde su punto de vista, ejerce sobre la decisión del retiro alguna de estas características frente a las demás. El capítulo 8 reseña fuentes bibliográficas sobre los sistemas de jubilación públicos y privados; se destaca el crecimiento sustancial que tales instituciones han registrado en todo el mundo habiéndose establecido "en 108 países a comienzos de 1975" (pág. 121); se analiza; en primer término, el comportamiento individual y agregado de una economía en respuesta a la seguridad social, en segundo lugar, se consideran los planes privados de jubilación ampliamente difundidos en las grandes compañías, a tal punto que llegan a cubrir aproximadamente la mitad de la mano de obra en EE. UU.; finalmente, se resumen los problemas con los que se enfrenta la formulación de las políticas de retiro: aumento de la cohorte de personas ancianas, hecho que incrementa la dependencia, movimientos migratorios e inflación. En el capítulo 9 se discuten estudios dedicados al problema del envejecimiento desde un enfoque macro'económico; en este sentido, se considera el impacto económico que un sistema registra en relación con un cambio en la estructura por edades; tal cambio se analiza considerando la productividad, la movilidad de la mano de obra, el consumo y el resto de las variables maeroeconómicas. En el capítulo 10, los autores, a modo de conclusión, destacan que el envejecimiento tiende a acentuarse sea por un tasa de fertilidad insuficiente para contrarrestar la de mortalidad sea por la posibilidad de una "revolución gereontológica"; presentan con cierto detalle, el análisis sobre el problema de la provisión de un adecuado sistema de seguridad social para la ancianidad y proponen nuevas líneas de investigación sobre el tema; por fin, instan a que se le preste mayor atención que la que ha recibido hasta el presente.

No escapará al economista dedicado a la economía de los recursos humanos el valor bibliográfico inapreciable que tiene la obra que aquí reseñamos al poner a su alcance el material más relevante y actual sobre el problema del envejecimiento de una población; entre dicho material podemos encontrar varios trabajos de los mismos autores. La contribución de Clark y Spengler cobra aún mayor dimensión si se considera que si bien algunos aspectos están trata- 
dos a nivel interdisciplinario, la temática fundamental del problema del envejecimiento demográfico se halla delimitado de manera rigurosa. Es oportuno señalar, sin embargo, que a veces se dan como sobreentendidos algunos supuestos que, a nuestro criterio, debieran haberse presentado de manera explícita como ocurre, por ejemplo, con la hipótesis sobre el estado estacionario de una población. Sorprende además, el lugar en que se presenta un análisis formalizado sobre el fenómeno de la dependencia; en efecto, el tema aparece recién en la conclusión mientras que hubiera sido más procedente abordarlo en el capítulo 3 que trata específicamente el problema del envejecimiento y su relación con la dependencia. Por supuesto que tales consideraciones en nada ensombrecen el nivel del trabajo. Es de desear que la valiosa aportación que representan las publicaciones de esta naturaleza sigan apareciendo de manera sistemática.

E. O. de Guevara

Lamas. ERnesto Raul. Un eficaz control estatal, Buenos Aires, Macchi, 1980, págs. 328.

Este libro trata uno de los temas al cual no se le ha dado la importancia que necesita: el control de la función pública. Las funciones que cumple el Estado se han incrementado hasta llegar a la intervención directa y amplia dentro de la economía para lograr los fines de política económica con el menor costo social. Pero los gobiernos deben dar cuenta de sus actos a quienes le han delegado el poder, surgen así las distintas responsabilidades de los funcionarios.

Su autor, el Dr. E. Lamas, ha desempeñado cargos de jerarquía en la Administración Pública y en la docencia tanto a nivel nacional como internacional. Es autor además de libros y publicaciones sobre los problemas que afectan a la función pública.

El libro consta de dos partes. La primera consiste en un ensayo presentado en 1966 en el concurso abierto del Segundo Congreso Latinoamericano de Entidades Fiscalizadoras. Los capítulos iniciales ofrecen una amplia información de control estatal, de los organismos que lo realizan, así como de las características más típicas de los sistemas de control aplicados por algunos países europeos y americanos, entre ellos nuestra administración. El autor critica el tipo de control realizado, que "se ejerce como un fin en sí mismo y no como un medio de verificar si se ha actuado de acuerdo al presupuesto". Esas deficiencias han sido criticadas - y se han considerado soluciones para perfeccionar los sistemas de control- en entidades o reuniones científicas citadas detalladamente por el autor. Las crecientes actividades que han asumido los Estados modernos requieren un control más dinámico, ampliándose el control tradicional de legitimidad o legalidad con un control de mérito o eficiencia de la acción cumplida, lo que el autor considera como un necesario control de los actos de la Administración Pública, frente a los controles existentes (interno y externo) que por sus procedimientos demasiado formales entorpecen la acción de la Administración. Esta primera parte culmina con una propuesta acerca de "las bases de ... un ágil, útil y responsable sistema de control complementario de la acción y administración estatal". Es así que se propone mantener el control de legitimidad y eficiencia aún cuando en forma simplificada, e instituir paralelamente un sistema conjunto de planificación y presupuesto por programa, y un control de eficiencia y productividad para evaluar el mérito de los organismos y funcionarios. También se indica la necesidad de superar la superposición de funciones entre organismos de control y se sugiere un racional deslindamiento de competencias, esquematizado en un cuadro de ordenamiento.

En la segunda parte, escrita posteriormente, el autor examina la evolución del control durante 1966-79, a la luz de las consideraciones expuestas en la primera. Basándose en la expe- 